\title{
Over-spinning a black hole with a test body
}

\author{
Ted Jacobson* and Thomas P. Sotiriou*† \\ * Center for Fundamental Physics, University of Maryland, College Park, MD 20742-4111, USA and \\ ${ }^{\dagger}$ Department of Applied Mathematics and Theoretical Physics, Centre for Mathematical Sciences, \\ University of Cambridge, Wilberforce Road, Cambridge, CB3 OWA, UK
}

(Dated: November 5, 2018)

\begin{abstract}
It has long been known that a maximally spinning black hole can not be over-spun by tossing in a test body. Here we show that if instead the black hole starts out with below maximal spin, then indeed over-spinning can be achieved when adding either orbital or spin angular momentum. We find that requirements on the size and internal structure of the test body can be met as well. Our analysis neglects radiative and self-force effects, which may prevent the over-spinning.
\end{abstract}

PACS numbers: 04.70.Bw Classical black holes, 04.20.Dw Singularities and cosmic censorship

Gravitational collapse in general relativity inevitably leads to spacetime singularities [1, 2] where the theory presumably breaks down. According to the "Cosmic Censorship" conjecture [3], "naked singularities" — i. e. singularities visible from afar — cannot be formed via any process that involves physically reasonable matter. Although this hypothesis remains unproven, significant evidence suggests that it may indeed be true, in the sense that whenever singularities arise from generic, nonsingular initial data, they may be always hidden behind a black hole event horizon (and may even be invisible to any observer) [4, 5]. One way of testing Cosmic Censorship is thus to ask whether it may be possible to destroy a black hole horizon.

The general stationary, vacuum black hole solution in general relativity is a Kerr black hole, which is characterized by its mass $M$ and angular momentum $J$. For angular momentum greater than the "extremal" limit $G M^{2} / c$, the Kerr metric has no horizon and has a naked singularity. The question we are posing is whether an object with either orbital or spin angular momentum - hereafter called the "body" - can be dropped into a Kerr black hole so as to push the resulting composite object over the extremal limit. If so, that object could not settle down to a stationary black hole. Presumably it would form a naked singularity, in violation of Cosmic Censorship.

This question was considered and answered in the negative long ago by Wald [6] under the assumptions that the black hole is initially extremal and the body can be treated as a test particle. Here we relax the first assumption, considering an initial black hole that is nearly but not quite extremal, and examining whether one can "jump over" the extremal limit in the test body approximation. Our question is motivated by Hubeny's analysis [7] which showed in an analogous setting that a charged test particle can be added to a charged black hole in such a way that the resulting object has too much charge to be a black hole, and by the analysis of Hod [8] which showed that a point test particle can be dropped from the horizon into a spinning black hole in such a way that the resulting object has too much angular momen- tum to be a black hole.

We extend Hod's result, finding a broader class of trajectories by which over-spinning may be accomplished, and we also consider physical structure and size limitations of the particle. It turns out that the latter do not preclude the over-spinning. Radiation and/or self-force effects, which lie outside the test body approximation, might nevertheless prevent the over-spinning. We note that the considerations of the present paper are strictly classical, in contrast to a number of recent studies of quantum mechanical tunneling processes that might be able to over-spin a black hole (see [9], and references therein). Hereafter we adopt units with $G=c=1$.

In order to be able to treat the body as a test body, we shall assume its energy $\delta E$ and angular momentum $\delta J$, defined with respect to the Killing vectors of the black hole, are small compared to those of the black hole,

$$
\delta E \ll M, \quad \delta J \ll J
$$

Note that the black hole must start out very close to extremal if the small perturbation caused by the body is to have any chance of pushing it over the extremal limit.

We assume that the angular momentum of the body is aligned with the spin of the black hole, in order to maximize the angular momentum added. The question, then, is whether $\delta E$ and $\delta J$ can be chosen such that the body falls into the black hole, with negligible corrections to the test body approximation, and such that

$$
J+\delta J>(M+\delta E)^{2} .
$$

This sets the lower bound on the required angular momentum carried by the body, for a given $\delta E$ :

$$
\delta J>\delta J_{\min }=\left(M^{2}-J\right)+2 M \delta E+\delta E^{2} .
$$

Since we are assuming $\delta E \ll M$, it might seem that the $\delta E^{2}$ term may as well just be neglected at this stage, but in fact that term imposes an upper bound on $\delta E$ and $\delta J$.

Equation (3) yields

$$
\delta J / \delta E^{2}>2 M / \delta E \gg 1
$$


If $\delta E$ were equal to the rest mass of the body, and if $\delta J$ comes from spin, this would imply that the body has angular momentum far over the extremal ratio. In itself this is not a problem, since there is no a priori upper limit to this ratio if one is not restricting to black holes. Note also that $\delta E$ can be much less than the rest mass, if the body is deeply bound.

An upper bound on the angular momentum of the body comes from the requirement that it does indeed cross the horizon to end up in the black hole. Wald [6] derived such a bound by analyzing the trajectories using the geodesic or Papapetrou equations for the case of orbital angular momentum and spin respectively. We will use another path to the same results which does not actually require the geodesic or Papapetrou equations.

If the body falls across the horizon, then the flux of energy and angular momentum into the black hole are related to the stress energy tensor of the body via

$$
\delta E-\Omega_{H} \delta J=\int T_{a b} \chi^{a} d \Sigma^{b} .
$$

Here $\Omega_{H}=a / 2 M r_{+}$is the angular velocity of the horizon, where $a=J / M$ the specific angular momentum and $r_{+}=M+\left(M^{2}-a^{2}\right)^{1 / 2}$ is the horizon radius in BoyerLindquist coordinates. The vector $\chi^{a}=\partial_{t}^{a}+\Omega_{H} \partial_{\phi}^{a}$ is the horizon-generating Killing vector, and $d \Sigma^{b}$ is the horizon surface element. Both $\chi^{a}$ and $d \Sigma^{b}$ are parallel to the null generator of the horizon, so the null energy condition on the matter of which the body is composed implies

$$
\delta E>\Omega_{H} \delta J
$$

which can be written as

$$
\delta J<\delta J_{\max }=\frac{2 M r_{+}}{a} \delta E .
$$

This constraint guarantees that the body can fall across the horizon starting from some point outside, although in general it is in a bound orbit that does not come from spatial infinity.

As long as $\delta J_{\min }<\delta J_{\max }$ for some $\delta E$, there will be values of $\delta J$ satisfying both inequalities (3) and (7). If the black hole starts out extremal $\left(J=M^{2}\right.$, so $\left.a=M=r_{+}\right)$, then $\delta J_{\text {min }}=2 M \delta E+\delta E^{2}$ is never less than $\delta J_{\max }=2 M \delta E$, so it is impossible to over-spin the black hole. This was observed long ago by Wald 6]. In the sub-extremal case, however, these inequalities can be satisfied.

To understand the nature of the allowed range it is helpful to visualize the inequalities graphically. If $\delta J_{\max }$ and $\delta J_{\min }$ are plotted vs. $\delta E$, the former is a straight line through the origin, with slope $2 M r_{+} / a>2 M$, while the latter is a parabola with positive intercept, slope $2 M$ at the intercept, and curved upwards. Some algebra reveals that the parabola always intersects the straight line in two points. The allowed values of $\delta E$ and $\delta J$ are those in the compact region above the parabola and below the straight line. Note that if the $\delta E^{2}$ is neglected in (3), the parabola is replaced by a straight line, and no upper bound is imposed on the allowed values. The case considered by Hod [8], i.e. that of dropping the particle from a point on the horizon, corresponds to the upper boundary of this region, $\delta J=\delta J_{\max }$.

Rather than giving exact formulae, it is more illuminating to expand in the small dimensionless quantity $\epsilon \ll 1$ defined by

$$
J / M^{2}=a / M=1-2 \epsilon^{2} .
$$

(Hubeny 4] used the same parameter to analyze the charged case.) Also at this stage we adopt units with $M=1$, which will keep the expressions simpler. Then we have

$$
\begin{aligned}
& \delta J_{\min }=2 \epsilon^{2}+2 \delta E+\delta E^{2} \\
& \delta J_{\max }=(2+4 \epsilon) \delta E,
\end{aligned}
$$

where terms of order $O\left(\epsilon^{2} \delta E\right)$ have been dropped in (10). The allowed range of $\delta E$ lies where the difference

$$
\delta J_{\max }-\delta J_{\min }=4 \epsilon \delta E-\delta E^{2}-2 \epsilon^{2}
$$

is positive, i. e.

$$
(2-\sqrt{2}) \epsilon<\delta E<(2+\sqrt{2}) \epsilon
$$

In particular, $\delta E$ must be of order $\epsilon$, which is consistent with the requirement (11) that the body make only a small perturbation. For a given $\delta E$, the allowed values of $\delta J$ are near $2 \delta E$, so we must have

$$
\delta J \sim \delta E
$$

Note that the width (11) of the allowed range of $\delta J$ is only of order $\epsilon^{2} \ll \epsilon$.

As already mentioned, the black hole must start out very nearly extremal, but now we can be somewhat more quantitative. According to (11) and (12) we must have $\epsilon \ll 1$, and $a-1=2 \epsilon^{2}$ is parametrically smaller. For example, if $\epsilon=10^{-2}$, then the initial black hole must have $a=0.9998$. For a thought experiment we can imagine even smaller values of $\epsilon$. We conclude that, if the body can be treated as a point test particle, the black hole can indeed be over-spun.

We turn now to consideration of the finite size requirements for the body, beginning with the case of orbital angular momentum in the equatorial plane. Here the issue is that in order to have the required values of $\delta E$ and $\delta J$, the body might have to be in a bound orbit, which would have a turning point at a maximum radius. In that case we would need to require that the body be small enough to fit outside the horizon at this radius. Since the body can be no smaller than a black hole with the same rest mass, it is not clear in advance whether 
this requirement could be met. However, we find that this size constraint is not an issue, since in fact there are orbits that come in from infinity with no turning point.

To address this point we recall that the proper time derivative of the (Boyer-Lindquist) radial coordinate of orbital motion in the equatorial plane satisfies $\dot{r}^{2} / 2+$ $V_{\text {eff }}(r, \tilde{E}, \tilde{L})=0$, where the effective potential is given in terms of the energy $\tilde{E}$ and angular momentum $\tilde{L}$ per unit mass by [10]

$$
V_{\mathrm{eff}}=-\frac{1}{r}+\frac{\tilde{L}^{2}}{2 r^{2}}-\frac{(\tilde{L}-a \tilde{E})^{2}}{r^{3}}+\frac{1}{2}\left(1-\tilde{E}^{2}\right)\left(1+\frac{a^{2}}{r^{2}}\right) .
$$

The turning points are located where $V_{\text {eff }}(r)=0$. For the energy $\delta E$ we choose the value at the center of the allowed region given above, so $\tilde{E}=2 \epsilon / m$, where $m$ is the rest mass of the body. The allowed range of the specific angular momentum is then $(2+3 \epsilon) \tilde{E}<\tilde{L}<(2+4 \epsilon) \tilde{E}$, so we are led to parametrize the specific angular momentum as $\tilde{L}=(2+b \epsilon) \tilde{E}$, where $3<b<4$. If values of $\epsilon, m$ and $b$ can be found for which $V_{\text {eff }}<0$ everywhere outside the horizon, then with such values a body can fall all the way into the black hole from infinity.

We explored this question numerically and found that such orbits indeed exist. Two examples with $\epsilon=0.01$ and $b=3.3$ are $m=0.01(\tilde{E}=2)$ and $m=0.001$ $(\tilde{E}=20)$. To understand a bit more quantitatively we can focus on large asymptotic velocity $\tilde{E} \gg 1$, dropping $\tilde{E}$ independent terms and expanding the potential out to second order in $\epsilon$, which yields

$$
\begin{aligned}
V_{\mathrm{eff}}=-\frac{\tilde{E}^{2}}{2}[1 & -\left(3+4 b \epsilon+\left(b^{2}+4\right) \epsilon^{2}\right) r^{-2} \\
& \left.+\left(2+4 b \epsilon+2\left(b^{2}+4\right) \epsilon^{2}\right) r^{-3}\right] .
\end{aligned}
$$

This potential is negative at $r=0$ and at $r=\infty$, and has a single maximum at $r=1+2 b \epsilon / 3+O\left(\epsilon^{2}\right)$, where it is equal to $-\left(2-b^{2} / 6\right) \tilde{E}^{2} \epsilon^{2}+O\left(\epsilon^{3}\right)$. Hence it is everywhere negative provided $b<\sqrt{12} \simeq 3.46$, in which case the body falls across the horizon.

Next we examine whether requirements relating to the size and structure of the body can be met in the spinning case. For simplicity we assume that the body is dropped along the symmetry axis. We first consider the case when $\delta E \sim m$, and the body is not spinning relativistically, so its spin angular momentum is given by $\delta J \sim m v R \sim \delta E v R$, where $v$ is the surface velocity and $R$ is the equatorial radius. The condition $v<1$ then implies $R>\delta J / \delta E$. We saw above that the ratio $\delta J / \delta E$ must be of order unity (13), that is of order $M$. In this case the body must be larger than the black hole, so it simply will not fit in the transverse direction, and in any case treating it as a point particle with spin would be unjustified, since that rests on the smallness of the size of the body compared to the ambient radius of curvature. Moreover, the radial tidal stress required to hold the body together would be larger than the energy density, violating energy conditions.

It cannot help to allow ultra-relativistic tangential velocity. The reason can be seen with a simple Newtonian estimate. If the surface acceleration $v^{2} / R$ exceeds $\sim 1 / R$, the required force exceeds $m / R$. But what could provide this force to hold the body together? The selfgravitational force $\sim m^{2} / R^{2}$ cannot produce this acceleration unless $m / R>1$, which is the condition that the body becomes a black hole. A black hole cannot satisfy (4), so this will not do. Alternatively, suppose the force is provided by internal tension. Then the $T_{\hat{r} \hat{r}}$ stress tensor component in an orthonormal rest frame must satisfy $-T_{\hat{r} \hat{r}} R^{2}>m / R$, hence $-T_{\hat{r} \hat{r}}>m / R^{3}$. But the right hand side is the energy density of the body, so this inequality violates all the energy conditions. The body would therefore need to be composed of unphysical material. The conclusion is that it is impossible to over-spin the black hole if the body's Killing energy is close to its rest mass, $\delta E \sim m$.

Since the angular momentum involves the rest mass $m$, not the Killing energy, it might be possible to achieve a large enough $\delta J$ with a small enough size $R$, without requiring unphysical matter, by dropping the body from a position where it is deeply bound, $\delta E \ll m$. This might be achieved by slowly lowering the body on a tether, down to the near the black hole horizon, before dropping it in. Now we reconsider whether the size restrictions can be met in this setting.

We begin with the restrictions on the rest mass $m$. If $m$ is much greater than $\delta E$, then the test body approximation requires that we impose not only $\delta E \ll 1(=M)$ (11), but also $m \ll 1$. There is also a lower bound on $m$, coming from an upper bound on $R$ : the angular momentum is $\delta J \sim m v R$, hence (restricting to nonrelativistic spin $v<1$ as required by the previous analysis) $R>\delta J / m \simeq 4 \epsilon / m$. The requirement $R \ll 1$ then yields $m \gg \epsilon$. The mass and size must therefore fall within the ranges

$$
\epsilon \ll m \ll 1, \quad 4 \epsilon / m \lesssim R \ll 1 .
$$

The inequality (6) guarantees that the body can cross the horizon with the chosen values of energy and angular momentum, but since the deeply bound drop point lies at a finite distance from the horizon it is necessary to check that (a) the spinning body would actually fall into the black hole rather than being repelled, and (b) the body polar radius, $R_{\text {polar }}$, can be chosen smaller than the proper distance $d$ from the horizon to the drop point

$$
R_{\text {polar }}<d,
$$

so that the body can fully fit outside the black hole and be localized at the drop point.

To address these issues, we note 11] that a spinning body of rest mass $m$, moving along the axis of rotation, 
has energy $\delta E$ and angular momentum $\delta J$ satisfying

$$
\delta E-\Omega(r) \delta J=m \gamma(r) N(r)
$$

where $\gamma(r)$ is the gamma factor for the body relative to the rest frame of the timelike Killing vector, and

$$
N(r)=\sqrt{\frac{r^{2}-2 r+a^{2}}{r^{2}+a^{2}}}, \quad \Omega(r)=\frac{2 a r}{\left(r^{2}+a^{2}\right)^{2}}
$$

respectively are the norm of the Killing vector (which vanishes at the horizon) and the limiting angular velocity of ZAMOs (zero angular momentum stationary observers) as the axis of rotation is approached. The body can exist at rest at radial coordinate $r$, with the given values of the constants of motion, if (18) is satisfied with $\gamma(r)=1$. The body will fall in across the horizon from such a point provided (i) the left hand side of (18) is positive at the horizon, i.e. (6) holds, and (ii) $r$ is the closest radius to the horizon where (18) is satisfied with $\gamma(r)=1$.

To estimate the maximum distance from the deeply bound drop point to the horizon, for a given mass $m$, we make use of the fact that $N \ll 1$ there. In this regime, and for a near-extremal black hole, $\Omega(r) \simeq$ $\frac{1}{2}-\sqrt{\epsilon^{2}+N^{2} / 2}$, and so for the required values of energy and angular momentum we have $\delta E-\Omega(r) \delta J=$ $O\left(\epsilon^{2}\right)+O(\epsilon N)$. This must be equal to $m N$, which according to (16) must be $\gg \epsilon N$. It follows that $\epsilon \gg N$, hence the horizon value $\Omega_{H} \simeq \frac{1}{2}-\epsilon$ may be used for $\Omega(r)$. Using $\delta J_{\min }(9)$ we find $\left(\delta E-\Omega_{H} \delta J\right)_{\max }=\epsilon^{2}$, (which occurs for $\delta E=2 \epsilon$ ). On the other hand, near the horizon we have $N \simeq \kappa d \simeq \epsilon d$, where $\kappa$ is the surface gravity (equal to $\lim _{r \rightarrow r_{+}}|\nabla N|$ ) and $d$ is the proper distance to the horizon (orthogonal to the surfaces $N=$ const). Hence the maximum value $d$ can take is $d \simeq \epsilon / m$. According to (16) we must therefore have $d \ll 1$, which justifies having used of the linear near-horizon approximation to $N$ [15]. To fit outside the horizon at the drop point, the body must therefore satisfy

$$
R_{\text {polar }}<\epsilon / m \text {. }
$$

This bound and the condition (16) on the equatorial radius $R$ can be simultaneously satisfied, provided the body is at least somewhat oblate, with $R>4 R_{\text {polar }}$. (For an earlier appeal to oblate bodies in this context see [14]).)

To summarize, we have considered gedanken experiments where an object with spin or orbital angular momentum is dropped into a near-extremal Kerr black hole, in an effort to drive the black hole beyond the extremal limit. We have found that, to the extent that radiative and self-force effects can be neglected, the black hole can be over-spun. In the orbital case this can be achieved even with an object dropped from infinity. In the spin case, the requirement that the internal stresses satisfy the energy conditions implies that the object has to be deeply bound and somewhat oblate.
We saw that, from purely kinematic considerations, the relation between the energy and angular momentum of the dropped body must be very finely tuned: they are both of order $\epsilon$ in magnitude, but the allowed window for angular momentum, given the energy, is only of order $\epsilon^{2}$. Since the over-spinning process we found involves a delicate balance, it is certainly possible that, although small, gravitational radiation and/or self-force [16] effects may always manage to preclude the over-spinning. Indeed, given the existing evidence for Cosmic Censorship, this seems likely. Our analysis suggests a dynamical regime in which it may be interesting to study these effects.

As a final remark, we note that if over-spinning can be achieved, then the likely formation of a naked singularity could provide an escape from Hawking's proof of the second law of black hole mechanics [12], that the black hole horizon area cannot decrease (or disappear), as well as from Israel's proof of the third law 13], that the surface gravity cannot be reduced to zero in a finite time.

We thank E. Barausse, A. Buonanno, S. Liberati, and E. Poisson for helpful discussions. This research was supported in part by the NSF under grant PHY0601800, and T. P. S. was also supported by STFC.

Note: The present version of this paper incorporates the oblate case correction which appears with the published version only as an erratum.

[1] R. Penrose, Phys. Rev. Lett. 14, 57 (1965).

[2] S. W. Hawking and R. Penrose, Proc. Roy. Soc. Lond. A 314, 529 (1970).

[3] R. Penrose, Riv. Nuovo Cim. 1, 252 (1969) [reprinted as Gen. Rel. Grav. 34, 1141 (2002)].

[4] R. M. Wald, arXiv:gr-qc/9710068.

[5] R. Penrose, J. Astrophys. Astron. 20, 233 (1999) http://adsabs.harvard.edu/abs/1999JApA...20..233P.

[6] Wald, R., Ann. Phys. 82, 548 (1974).

[7] V. E. Hubeny, Phys. Rev. D 59, 064013 (1999).

[8] S. Hod, Phys. Rev. D 66, 024016 (2002).

[9] G. E. A. Matsas, M. Richartz, A. Saa, A. R. R. da Silva and D. A. T. Vanzella, Phys. Rev. D 79, 101502 (2009).

[10] R. M. Wald, General Relativity (Univ. Chicago Press, 1984).

[11] Y. Mino, M. Shibata and T. Tanaka, Phys. Rev. D 53, 622 (1996) [Erratum-ibid. D 59, 047502 (1999)].

[12] S. W. Hawking, Phys. Rev. Lett. 26, 1344 (1971).

[13] W. Israel, Phys. Rev. Lett. 57, 397 (1986).

[14] F. de Felice and Y. Q. Yu, Class. Quant. Grav. 18, 1235 (2001).

[15] More generally, in the near-horizon region $(N \ll 1)$ of a near-extremal black hole $(\epsilon \ll 1)$ one finds $N(r) \simeq$ $\sqrt{2} \epsilon \sinh (d / \sqrt{2})$.

[16] Hod [8] suggested that the effect of self-force can be estimated by making the replacement $\Omega_{H} \rightarrow \Omega_{H}+\omega \delta J$ in the bound (6), and noted that if $\omega>1 / 8$ this would suffice to preclude over-spinning. 